\title{
Correspondence
}

\section{The recruitment legacy of COVID-19}

With increasing thought being put into COVID-19 and the implications for psychiatric care in the months and years to come, it would be remiss to not consider the potential impact this could have on recruitment to the specialty.

Recruitment in psychiatry is a national, if not global problem, and has been for some time. Although frequently highlighted, there has been limited work clarifying the reasons behind the problem, which in turn has led to muted and potentially ineffective responses. This could all now be amplified by the COVID-19 crisis.

We know that foundation doctors already have very low exposure to psychiatry placements and teaching; the proportion of psychiatry teaching time has been found to be as low as $2.3 \%$ relative to surgical and medical specialties seeing $44.1 \%$. We also know that there is a significant correlation between trainees having a foundation placement in psychiatry and going on to apply for specialty training. ${ }^{1}$ This has been replicated in targeted studies with a surprisingly high $45 \%$ of psychiatric specialists found to not have even considered the specialty before their foundation years. $^{2}$

So, it is not an understatement that the current foundation year trainees are to be drastically affected. Not only have rotations been paused, but huge numbers have been redeployed. Already, the invaluable experiences of an entire cohort have been curtailed, with more likely to follow. Additionally, national exams have been cancelled; the impact of this and the choices made thereafter may even lead to very immediate-term shortages and unknown ramifications.

This is before we even consider those that will shortly follow them through, the medical students. The proportionally enormous amount of time lost directly affects many of the clearly identified factors attracting students to the field such as placement and elective exposure. ${ }^{3}$ There have recently been some targeted strategies implemented by the Royal College of Psychiatry to try and improve recruitment - especially the 'Choose Psychiatry' campaign - these outreach programmes might serve to be a much-needed lifeline more than ever before. Psychiatric societies within universities might be the great untapped resource that prevail the shutdown and educational restrictions. ${ }^{4}$ Could they, in turn, be a vital commodity?

There is a growing recognition that although it may appear to be minutiae relative to the scale of a global pandemic, COVID-19 could have a profound effect on the career progressions of a generation of doctors. ${ }^{5}$ Only by addressing this early and actively can we mitigate potential disaster for years to come.

Rajaei K. Sharma, Medical Student, University of Exeter, UK; email: rajaei. sharma@nhs.net; Bhupinder Sharma, Consultant, Royal Marsden Hospital, UK NICE Expert Advisor Centre for Guidelines Development, UK; Harriet L. Ogle, Medical Student, University of Exeter, UK

\section{Declaration of interest}

1 Choudry A, Faroog S. Systematic review into factors associated with the recruitment crisis in psychiatry in the UK: students', trainees' and consultants' views. BJPsych Bull 2017; 41: 345-52.

2 Doris M, Grimason A, Hughes D, O'Neill E, Parks L, Carragher A. Who wants to be a psychiatrist? Northern Ireland foundation doctors (20062018) are positive toward psychiatry as career choice. BJPsych Bull [Epub ahead of print] 31 Jan 2020. Available from: http://doi.org/10.1192/bjb. 2019.86

3 Farooq K, Lydall G, Malik A, Ndetei D, Bhugra D. Why medical students choose psychiatry - a 20 country cross-sectional survery. BMC Med Educ 2014; 14: 12.

4 Pandian H, Mohamedali Z, Chapman G, Vinchenzo P, Ahmed S, Mulliez Z, et al. Psych Socs: student-led psychiatry societies, an untapped resource for recruitment and reducing stigma. BJPsych Bull 2020; 44: 91-5.

5 Ferrel M, Ryan J. The impact of COVID-19 on medical education. Cureus 2020; 31: 3 .

doi:10.1192/bjb.2020.63

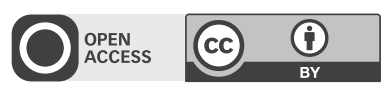

(c) The Author(s) 2020. This is an Open Access article, distributed under the terms of the Creative Commons Attribution licence (http://creativecommons. org/licenses/by/4.0/), which permits unrestricted re-use, distribution, and reproduction in any medium, provided the original work is properly cited.

\section{Highlighting some of the challenges COVID-19 has posed to the European Convention on Human Rights}

The European Convention on Human Rights (ECHR) came into force in 1953 to guarantee specific rights and freedoms for people in countries that belong to the Council of Europe and to protect their human rights and prohibit unfair and harmful practices. ${ }^{1}$

COVID-19 took the world by storm and invaded all aspects of humanity. The 'normal approach' we had to life and issues relating to everyday living changed. New norms/ways of thinking as well as laws ${ }^{2}$ emerged to tackle the sweeping public health crisis with consequent implications for accepted rights and freedoms under the ECHR.

Governments' world over (including Europe) variously imposed lockdown measures to limit the spread of the disease and subsequently introduced schemes to alleviate the financial difficulties imposed on their populace and pioneered schemes such as volunteer services to help the less able with shopping, collecting medication from pharmacies etc, to lessen the hardship(s) imposed by lockdown measures.

The powers available to public authorities under Article 5 (1)(e) of the ECHR to lawfully detain people for the prevention of the spreading of infectious diseases were imposed by governments across Europe with the consequence that health and law enforcement agencies acquired powers to confine otherwise healthy individuals to their homes and to isolate and screen individuals suspected to have contracted COVID-19 using powers under Article 5(1)(b) of the ECHR. ${ }^{3}$ 